\title{
FastHVAC - A library for fast composition and simulation of building energy systems
}

\author{
Sebastian Stinner Markus Schumacher Konstantin Finkbeiner Rita Streblow \\ Dirk Müller \\ RWTH Aachen University, E.ON Energy Research Center, Institute for Energy Efficient Buildings and Indoor \\ Climate, Aachen, Germany \\ \{sstinner, mschumacher\} deonerc.rwth-aachen.de
}

\begin{abstract}
This paper describes the implementation of a Modelica library that is designed to enable fast composition and simulation of building heating, ventilation and air conditioning (HVAC) systems. The library is based on an approach which is focusing on the thermal behavior of the components. Compared to existing libraries, it has no information about the pressure of the system. This approach limits the applicability of the library, but decreases the computational effort as well as the time to set up a model. Nonetheless, the simulation results are comparable to simple HVAC system models based on Modelica.Fluid.
\end{abstract}

Keywords: HVAC, building performance simulation, building energy systems, city district simulation

\section{Introduction}

The dynamic simulation of building energy systems, including HVAC technologies, is gaining importance in recent years. Various open source libraries for the simulation of HVAC systems are available today (AixLib, Buildings, BuildingSystems, IDEAS) (Fuchs et al., 2015; Wetter et al., 2014; Nytsch-Geusen et al., 2013; Baetens et al., 2015) and will be unified in the context of the IEA EBC Annex 60 activities (Annex60, 2015; Wetter et al., 2015). The applications of the simulation models range from the rapid prototyping of new components and systems, over development of advanced control systems to reuse of models during operation for fault detection and diagnostics (Wetter, 2009).

In recent years, applications for BES system models have been extended to analyze entire city districts (Müller et al., 2015). This is especially important, when considering the interconnection of buildings, which can be in terms of district heating networks or the electrical grid. In this field, measures like Demand Side Management (DSM) (Müller et al., 2015) are expected to become more important in the future. The simulative analysis of these (large) energy systems with implemented thermal storages, as well as heat generators, e.g. heat pumps and combined heat and power plants, requires the analysis of large equation systems and might lead to unreasonable computational effort.

Furthermore, the simulation of (especially closed) hydraulic circuits, whose models are based on the package Modelica. F luid of the Modelica Standard Library (MSL) shows considerable difficulties. In particular, the steady-state initialization of these systems can be a critical issue. Singularities in the solution can arise. These singularities do not occur in single component testing, but in the composition of the closed hydraulic cycle. For inexperienced users, this behavior can be confusing (Casella, 2012). Even if the aforementioned phenomenon is encountered by experienced users, it might be a challenging task to set the initialization values properly. Especially in cases where the thermal investigation of energy systems is focused, it requires additional expenditure without benefit. Furthermore, the computational effort can be a critical factor, depending on the size of the observed system. The number of initialization variables can grow very fast (Casella et al., 2011). In case of a city district analysis, this becomes a very critical issue, as the building energy systems should probably be parameterized automatically with little manual input. As most of the critical problems in HVAC simulations arise due to pressure calculations, the authors developed an additional library for the simulation of building energy systems that takes into account the whole thermal behavior, while reducing the information about the hydraulic circuit to the mass flow rate. The introduced library is particularly suitable for applications such as rapid prototyping of new energy systems and the development of advanced control systems for heat generators.

In the following, we will introduce the approach that forms the basis for all simulation models in the presented library. First, all required equations and base classes are introduced. Afterwards, we give an overview of the library itself and the included packages with a selection of the models. The developed library enables the user 
for fast composition and simulation of building energy systems. However, the accuracy of the simulated models has to be verified. This is shown in chapter 4 by a comparison of a building energy system modeled with both the Modelica.Fluid components and the components of the new library, called Fast HVAC .

\section{Modelling principles}

The models of the FastHVAC library are designed based on standard mass and energy balances in individual components. Each component includes a volume, whose temperature, specific heat capacity (thus also enthalpy) and density is considered to be homogenous. To have the ability to interconnect different components, a new connector, that only transports the required information between the components, has been developed. This connector is called EnthalpyPort. It carries the following information:

- the mass flow rate in $\mathrm{kg} / \mathrm{s}$ : designed as a flow variable, from the connection point into the component

- the thermodynamic temperature in $\mathrm{K}$

- the specific enthalpy of the fluid in $\mathrm{J} / \mathrm{kg}$ as a stream variable

- the (constant) specific heat capacity of the fluid in $\mathrm{J} /(\mathrm{kg} \mathrm{K})$

Here, the specific heat capacity is a redundant information. It is needed for development purposes of the FastHVAC library. After completition of the development phase, the variable will be removed. With known temperature difference, mass flow rate and the specific heat capacity, it is possible to calculate the heat flow at each time step. Considering the component's thermal behavior, it facilitates the fault detection of models.

To make use of the aforementioned variables, balance equations for each component are formulated in accordance with Figure 1.

$$
\sum \dot{m}_{i}=0
$$

This equation defines that the fluid mass in the components does not change over time and entering mass flows have to leave through another connection. Furthermore, the energy balances are formulated:

$$
\begin{gathered}
c_{1}=c_{2}=c \\
T_{2}=\text { heatPort.T; } \\
h_{2}=c \cdot T_{2} \\
\dot{Q}=\dot{m} \cdot\left(h_{2}-h_{1}\right)
\end{gathered}
$$

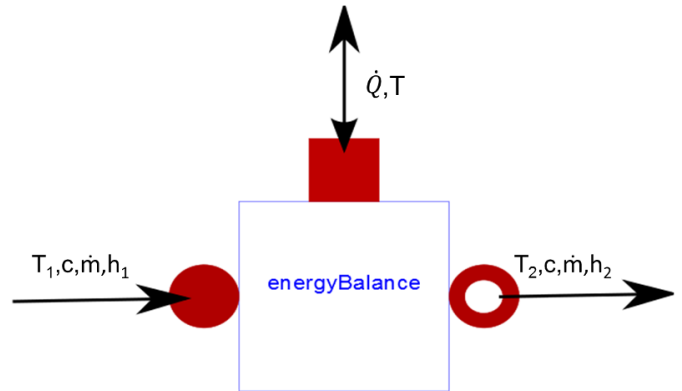

Figure 1. Exemplary scheme of the model EnergyBalance

The model EnergyBalance is based on the equations (1) to (5) and forms the basis for further components. Here, for entering flows index 1 is used and index 2 for outgoing flows, see Figure 1.

In case of hydraulic networks with multiple branches it is necessary to split off and merge the mass flows. Since there is no temperature change, the splitting of mass flows is described by the standard mass balance:

$$
\begin{gathered}
\dot{m}_{\text {in }}=\sum_{i=1}^{n} \dot{m}_{\text {out }, i} \\
T_{\text {in }}=T_{\text {out }} \quad c_{\text {in }}=c_{\text {out }} \quad h_{\text {in }}=h_{\text {out }}
\end{gathered}
$$

Merging the entering mass flows with different temperatures requires a calculation of the outgoing temperature. The temperature of the outgoing flow is obtained by balancing the mass and enthalpy flows:

$$
\begin{gathered}
\dot{m}_{\text {out }}=\sum_{i=1}^{n} \dot{m}_{\text {in }, i} \\
\dot{H}_{\text {out }}=\sum_{i=1}^{n} \dot{H}_{\text {in }, i} \\
T_{\text {out }}=\frac{\dot{H}_{\text {out }}}{c_{p} \cdot \dot{m}_{\text {out }}}
\end{gathered}
$$

If a certain heat capacity is available in the component due to fluid content, the component HeatTransfer.Components.HeatCapacitor from the package Modelica. Thermal of the MSL, is introduced. Following this approach, we assure that the temperature dynamics within the component are properly represented and the leaving fluid has the actual component fluid temperature.

As many of the components (e.g. heat generators, pipes) have a capacity and need an energy balance as well, a combination of both systems is implemented forming the model WorkingFluid. This component is basically equivalent to the Vessels. ClosedVolume model of the Modelica.Fluid package within the MSL. The WorkingFluid model has a HeatPort_a connection to the outside, where heat can be injected or removed from the component. 
The commonly used fluid in building heating systems in Germany is water. However, within the operating range of standard systems, the fluid properties underly only marginal changes. Therefore, we assume for the first version of the Fast HVAC library properties like density, specific heat capacity and thermal conductivity to be constant.

\section{Library for building HVAC systems}

\subsection{Packages of the FastHVAC library}

The FastHVAC library is organized into the packages shown in Figure 2. Most packages contain a package Baseclasses, which comprises essential models for the individual components.

$\begin{array}{ll}\text { FastHVAC.BaseClasses. } & \text { EnergyBalance } \\ \text {. WorkingFluid } \\ \text { FastHVAC.Components. HeatGenerators } \\ \text {.Storage } \\ \text {.Pipes } \\ \text {.Pumps } \\ \text {.Valves } \\ \text {.HeatExchanger } \\ \\ \text {.Sources } \\ \text {.Sinks } \\ \text { FastHVAC.Examples } & \\ \text { FastHVAC.Interfaces } & \\ \text { FastHVAC.Media } & \end{array}$

Figure 2. Package structure of the Fast HVAC library, current state. Only the major packages are shown.

\subsubsection{Package BaseClasses}

The package Baseclasses contains the models EnergyBalance and WorkingFluid, which represent the principles described in section 2 .

\subsubsection{Package Components}

The classes that form the core of the FastHVAC library can be found in the package components. These can be organized into components for generation, storage and distribution of thermal energy. Besides, there are additional packages that enable device control systems by measurements or are necessary to form proper energy balances at the system boundaries. In the following, these individual components will be briefly introduced.
- The generation of thermal energy can be represented by three different technologies, namely a Boiler, CHP or HeatPump component. Additionally, we include the model of a Solar. Thermal as a renewable source of heat production, which can be directly coupled to a test reference year (TRY) (DWD, 2011) based weather component.

- The model storage is the implementation of a stratified water storage tank with variable dimensions. The tank loading and unloading can occur either directly via mass flow injection or indirectly via up to two heating coils. The number of connection pairs for direct loading and unloading cycles is variable. The position of the connection pairs is variable as well and can be adjusted to individual purposes. A more detailed description and assessment of this component is shown in section 4.1.3.

- To cover the distribution of produced thermal energy to a room or building, further models are introduced. The model Pumps is used to generate a heating fluid mass flow through the system, whereas the model Pipes is used to direct the fluid to its destination and provide a physical connection to the ambient (e.g. air or soil). Since no pressure calculations are performed in the FastHVAC approach, the fluid mass flow rate is an external input to the Pump model and can be ideally set. Valves are needed to split off and merge fluid mass flows in multiple circuits. Due to the missing pressure information, the valve functionality is based on external signals. Considering a two-way valve, an equivalent mass flow rate to the valve opening can be calculated, e.g. by using a valve characteristics function. The calculated mass flow rate is used as the external input for the Pump model. If one closed loop with two branches is considered, a three-way valve can be used as well as two pumps inside each branch. The three-way valve divides the incoming mass flow rate into two flows. The ratio can be controlled by an external input. In case of more than two branches within one closed loop, a combination of three-way valves and pumps can be used.

- In cases where a conductive heat transfer has to be considered, e.g. in a domestic hot water (DHW) station, the model DHWHeatExchanger in the package HeatExchanger is applied. To supply the building with heat for space heating, a model for radiators called Radiator_ML is incorporated in the package HeatExchanger. Several parameters of the heat exchangers, for instance dimensions and discretization, can be adjusted.

- To enable the consideration of non-circular energy flows across the system boundaries, such as the flow of fresh water from public supply into the DHW 
system, Sources and Sinks are available within the library. These models are comparable to the Modelica.Fluid components.

- For device control purposes, measurement tools like a temperature sensor and a mass flow sensor are available within the package sensors .

\subsubsection{Other packages}

The package Examples contains example applications for each component that illustrate the typical use, test and validation cases. The package Interfaces contains connectors to build interconnections between individual components. This means especially the connector EnthalpyPort and the derived connectors. The package Media contains data records of different medium models.

\section{Example applications}

As the models created with the FastHVAC approach should not change the physical behavior of a considered system, we compare them to implementations with other models. For comparison purposes, each component is modeled based on Modelica.Fluid (HVAC component) as well as on the new approach (FastHVAC component). In the following, we will use the Modelica.Fluid based approach as reference.

First, different single components will be analyzed separately. In this case, the boiler, the radiator and the heat storage are shown. For each component, a Modelica.Fluid based model is compared to a FastHVAC model. For the boiler and the radiator, both models have exactly the same functionality and parameters. In the case of the heat storage, an open source model is used for the comparison. We use a model from the Buildings library (Wetter et al., 2014) for verification of the FastHVAC model. Afterwards, we will present the comparison results of a whole building energy system. It is a heat supply system for a dwelling, which comprises a heat consumer, heat generator, heat exchanger and a pipe network.

\subsection{Heat supply components for a dwelling}

For verification purposes, we will compare the dynamic thermal behavior of each component in simple test cases. The utilized medium record for Modelica.Fluid based components is the simple liquid water with constant properties (incompressible). The record values for FastHVAC.Media are identical to ConstantPropertyliquidWater data from Modelica.Media.Water within MSL (specific heat capacity, density, thermal conductivity). In the following, the different test cases are introduced.

\subsubsection{Test case: Boiler}

The heat generation is provided by a gas fired boiler. The boiler model is able to vary the flow temperature by modulating operation, e.g. to hold a certain flow temperature in case of varying return mass flow rate or return temperature. The observed time period is set to $1 \mathrm{~d}$ and the simulation resolution to $60 \mathrm{~s}$.

In this test case, the flow temperature and mass flow rate are constant while the heating power is increasing linearly from $30 \%$ to $100 \%$ within $10 \mathrm{~h}$. Furthermore, the boiler is switched off for $5 \mathrm{~h}$ after every $5 \mathrm{~h}$ of operation.

In Figure 3 the simulation results of both approaches are shown. The boiler return temperature is the variable of comparison. For this rather simple test case, the results are identically equal.

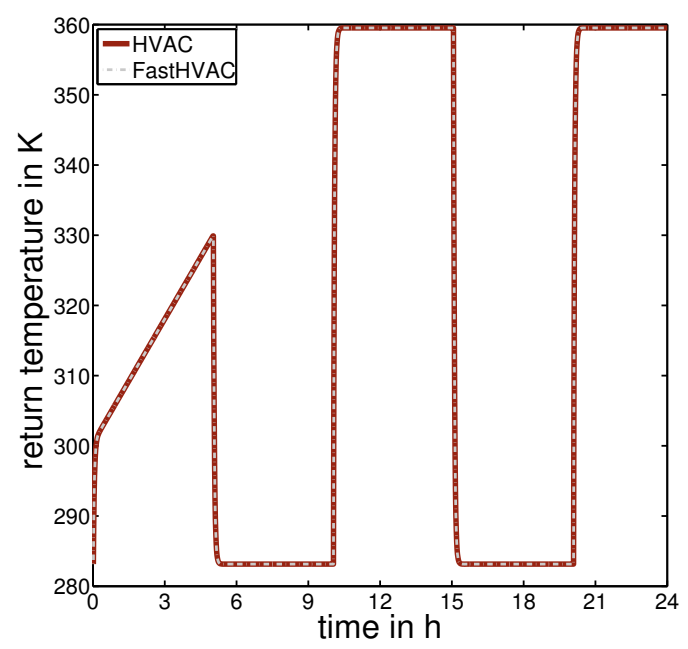

Figure 3. Boiler return temperature

\subsubsection{Test case: Radiator}

The heat distribution to the dwelling is provided by a radiator and is based on radiative and convective heat transfer. In this case, the thermal output of the radiator is determined by the building heating load. During nighttime, the heating load is higher than during daytime, mainly because of the absence of solar gains. In the considered case, the flow temperature signal is a sine wave with a period of $24 \mathrm{~h}$. This should emulate a dynamic operation of the system. The mass flow rate and ambient temperature are constant. The observed time period is again set to $1 \mathrm{~d}$ and the simulation resolution to $60 \mathrm{~s}$.

Target variables are the return temperature and the thermal output of the radiator. The comparative simulation shows identical results, see Figure 4.

\subsubsection{Test case: Heat storage}

A heat storage is not part of the closed heat supply system considered in section 4.2 , but it is an important 

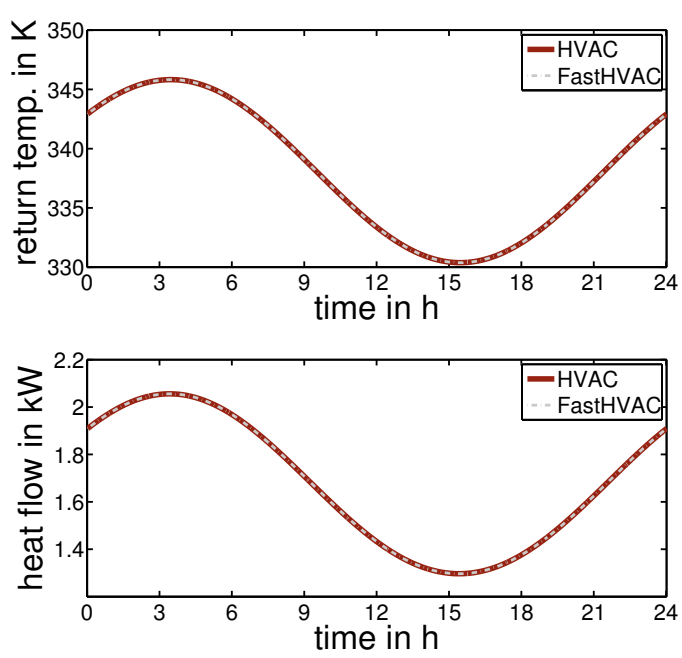

Figure 4. Return temperature and thermal output of radiator

component in general. For instance, in case of DSM, a thermal storage is necessary to decouple heat generation from heating demand. The heat transfer between the individual storage layers is based on conductance, buoyancy and enthalpy flow. Heat losses through the wall are accounted for as well.

The storage model Fluid.Storage. Stratified within the Buildings library is based on the same heat transfer principles (Wetter, 2015a). Therefore, we compare the storage following the FastHVAC approach, to the Buildings storage model for verification purposes. It is important to note that the storage wall construction of the considered models differs in its structure. In case of the model Buildings.Fluid.Storage. Stratified,

the whole storage wall is represented by the model Thermalconductor of the package Modelica. Thermal. Heat Transfer. Components. In contrast, the storage wall of the FastHVAC component consists of a tank wall and a tank insulation. Both, the wall and insulation are represented by a combination of the models ThermalConductor, HeatCapacitor of the package Modelica.Thermal. Heat Transfer. Components and additionally a model for heat convection. All other parameters and model details are identical. Both storage models are parameterized with the same dimensions, five layers and the same characteristical time constant for mixing. The ambient temperature is set constant.

The considered case shows a typical use case of a heat storage tank. The storage is first on standby mode followed by an alternating unloading and loading operation. This operation is modeled as a pulse signal, which is changing the flow temperature. The mass flow rate is modeled as a pulse signal as well. The amplitude changes between zero, in case of standby mode, and the nominal mass flow rate of the loading and unloading cycle.
Variables of interest are the top and bottom layer temperatures and the heat losses. Figure 5 shows the simulated dynamic temperatures of the top and bottom storage layer. The results are comparable, especially during the loading and unloading operation. For clarification, the temperature differences are shown in Figure 5 as well. The differences fade out when coming closer to a steady-state. The coefficient of determination is in both cases nearly 1 . Due to different wall constructions, the dynamic heat losses are different, as shown in Figure 6. However, the mean values are in a comparable range, with those of the Buildings component slightly higher.
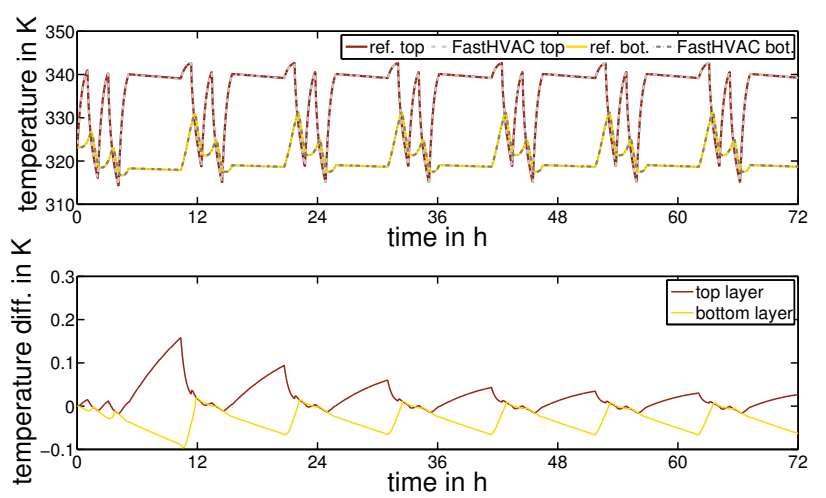

Figure 5. Absolute temperatures and temperature differences of top and bottom storage layers

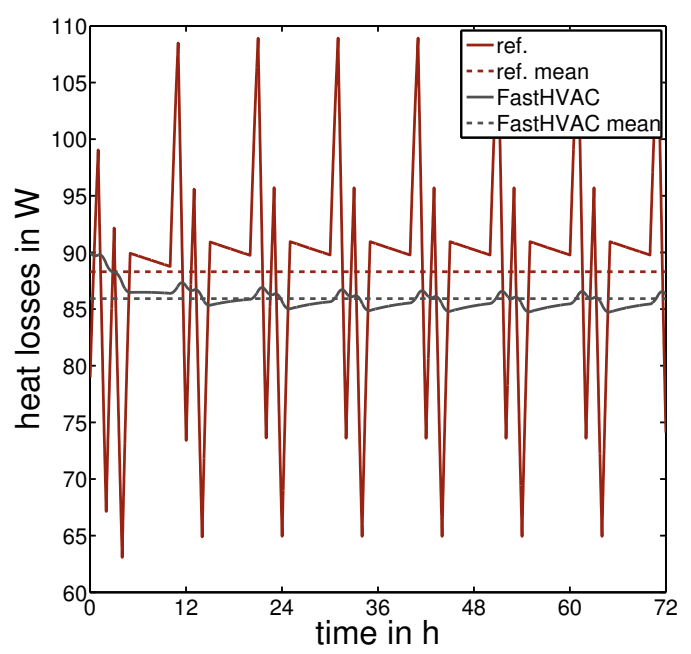

Figure 6. Storage heat losses to ambient

\subsection{Heat supply system for a dwelling}

The considered model of a dwelling heat supply system following the FastHVAC approach is shown in Figure 7. The models Boiler and Radiator_ML are already introduced. The dwelling as a heat consumer is represented by the model thermalzonewhich is freely available in the open-source model library AixLib (Fuchs et al., 2015). This model describes the thermal building behavior under given environmental conditions (Lauster et al., 
2014). In this case, we consider a single-family house with an insulation standard corresponding to the year 2002. The model weather represents the environment. Based on a TRY of the Germany's National Meteorological Service, weather data input signals (e.g. ambient air temperature, solar irradiation) are fed into the building model (DWD, 2011). The model PID controls the room temperature by varying the fluid mass flow through the boiler. The model controllerBoiler controls the radiator flow temperature by adapting the thermal output of the boiler.

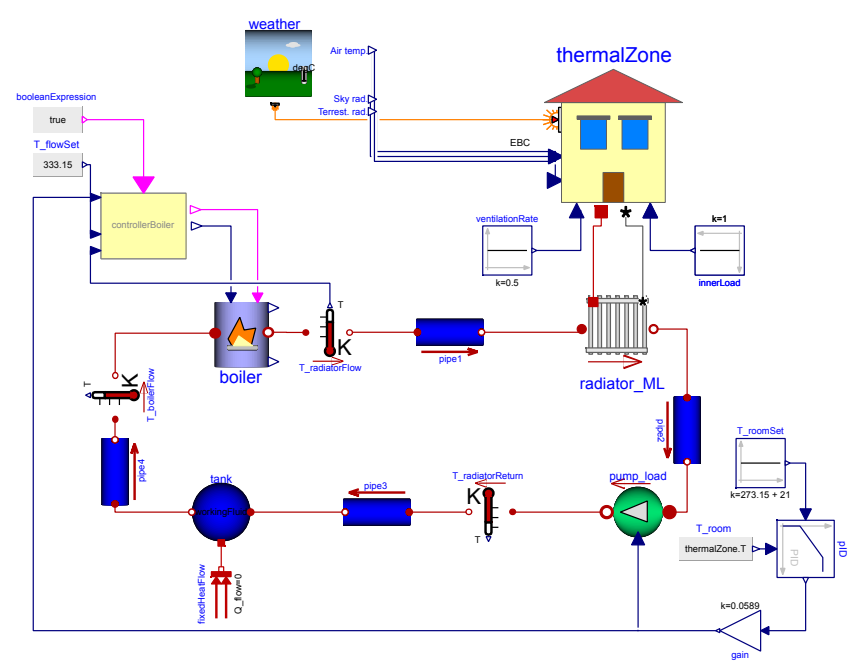

Figure 7. Model of heat supply system

In case of a closed thermo-hydraulic circuit with an incompressible fluid based on Modelica.Fluid, a pressure reference is necessary to avoid a structural singularity (Wetter, 2015b). For this reason, an adiabatic tank model is used within the Modelica.Fluid based variant. For closed thermo-hydraulic circuits, based on the new approach, these kind of measures are not necessary. However, for the observed case, it is considered for comparison purposes. The additional fluid content which is brought to the system by pipes and the tank has an influence on the dynamic behavior of this system. To take this fluid content into account, the models tank and pipe which contain the component workingFluid are applied. In order to achieve adiabatic conditions for these components, the heat transfer to the environment is set to zero or is disabled.

In this heat supply system, the room temperature is set to $294.15 \mathrm{~K}$. The radiator flow temperature is set to $333.15 \mathrm{~K}$. The simulated time period covers one year and the output resolution is $1 \mathrm{~h}$. Variables of interest are the boiler gas demand, the room temperature and the fluid mass flow.

In case of the fluid mass flow, the simulated results have no significant differences. Figure 8 shows a scatter plot of the dynamic fluid mass flow rate of both approaches. In the range of lower mass flow rates, there is a minor deviation. Nevertheless, the coefficient of deter- mination is approximately 1.

The deviation of the mass flow rates arises in cases of closed hydraulic circuits. The initial conditions of hydraulics in case of the Modelica.Fluid based variant (e.g. dp_small, m_flow_small, m_flow_start, etc.) have an influence on this deviation. The initial hydraulics settings cannot be set randomly due to the initialization difficulties mentioned in the introduction.

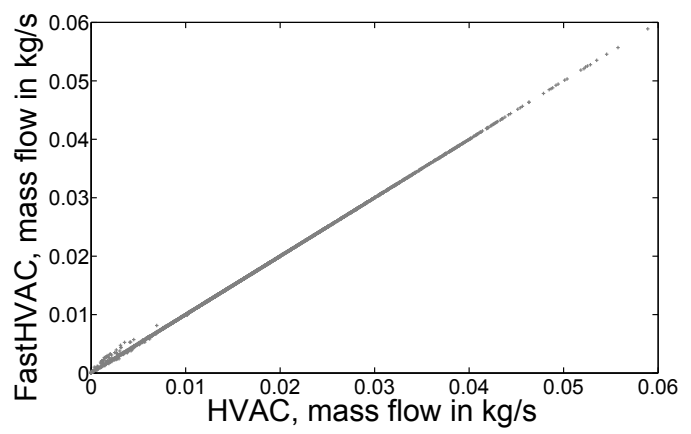

Figure 8. Comparison of fluid mass flow rates

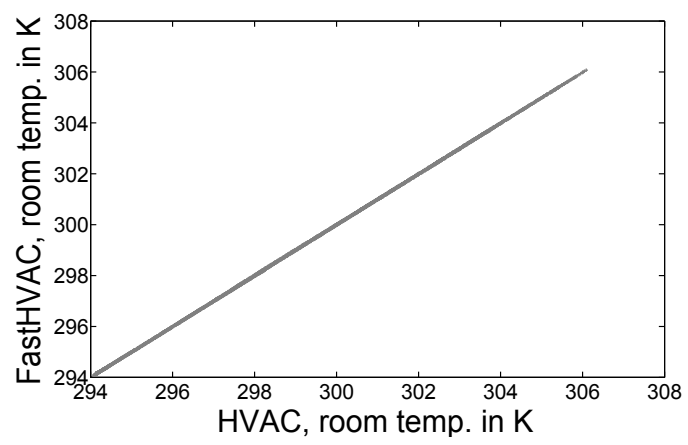

Figure 9. Comparison of room temperatures

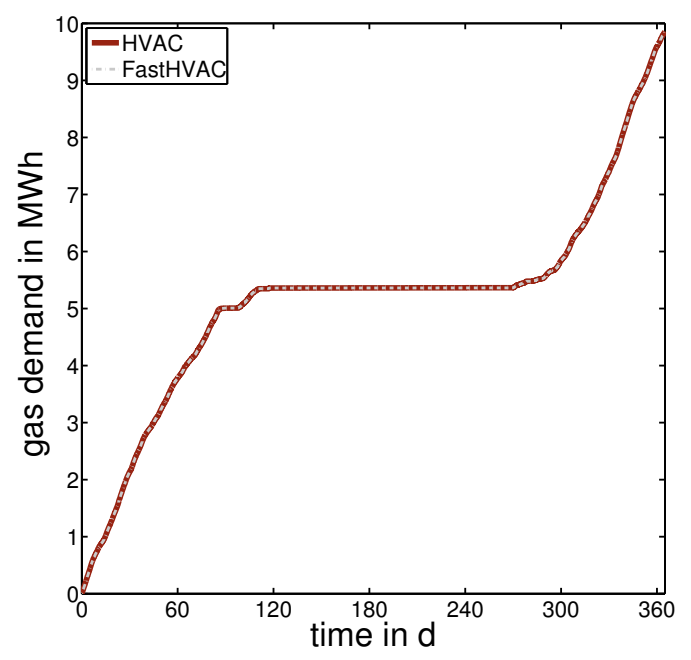

Figure 10. Boiler gas demand

Due to the minor deviation of the mass flow rate, the simulated dynamic room temperatures differ as well. A scatter plot of the simulation results is shown in Figure 
9. However, the coefficient of determination is approximately 1 . There is no active cooler inside the system. For this reason, the room temperature in the summer period is higher than the room set temperature.

The minor mass flow rate deviation has almost no influence on the boiler gas demand. Figure 10 shows the gas demand for the whole simulation period. In this case, the coefficient of determination is approximately 1 as well, with a negligible error for the annual gas demand.

Having a look at the simulation speed, we see a major improvement with the FastHVAC library. In case of the Modelica.Fluid based system, we found a computational effort of 379 seconds, compared to 79 seconds following the new approach.

\section{Conclusions and work in progress}

In this paper, we presented the design of a building HVAC library. This library is particularly suitable for applications such as rapid prototyping of innovative energy systems and the development of advanced control systems for heat generators. It is designed to decrease computational effort, while maintaining the result quality of more complex libraries. As stated in the case study, the simulation speed can be increased noticeably following our new approach. Nevertheless, the results are almost identical to more complex approaches, with coefficients of determination of approximately 1 for individual components as well as on system level. The modelling principle can be transferred to additional components. The presented models can be utilized to perform city district simulations. Thereby, measures like DSM can be evaluated.

In future work, we will extend the media representations to media with temperature dependent properties. Additionally, further components will be added, enabling the analysis of a greater variety of building and district energy systems.

\section{References}

Annex60. IEA EBC Annex 60 Modelica library, 2015. URL https://github.com/iea-annex60/ modelica-annex60.

Ruben Baetens, Roel De Coninck, Filip Jorissen, Damien Picard, Lieve Helsen, and Dirk Saelens. OpenIDEAS - an open framework for integrated district energy assessments. In Proceedings of the 14th IBPSA Conference, 2015. (submitted).

Francesco Casella. On the formulation of steadystate initialization problems in object-oriented models of closed thermo-hydraulic systems. pages 215-222. 2012. doi:10.3384/ecp12076215. URL http://www.ep. liu.se/ecp_home/index.en.aspx?issue $=76$.
Francesco Casella, Michael Sielemanny, and Luca Savoldelli. Steady-state initialization of object-oriented thermo-fluid models by homotopy methods. 2011. URL https://www.modelica.org/events / modelica2011/Proceedings/pages/papers/ 04_2_ID_131_a_fv.pdf.

Deutscher Wetterdienst DWD. Aktualisierte und erweitere Testreferenzjahre (TRY) von Deutschland für mittlere und extreme Witterungsverhältnisse. Technical report, Bundesinstitut für Bau-, Stadt- und Raumforschung, 2011.

Marcus Fuchs, Ana Constantin, Moritz Lauster, Peter Remmen, Rita Streblow, and Dirk Müller. Structuring the building performance Modelica model library AixLib for open collaborative development. In Proceedings of the 14th IBPSA Conference, 2015. (submitted).

Moritz Lauster, Jens Teichmann, Marcus Fuchs, Rita Streblow, and Dirk Mueller. Low order thermal network models for dynamic simulations of buildings on city district scale. Building and Environment, 73:223-231, 2014. ISSN 03601323. doi:10.1016/j.buildenv.2013.12.016.

Dirk Müller, Antonello Monti, Sebastian Stinner, Tim Schlösser, Thomas Schütz, Peter Matthes, Henryk Wolisz, Christoph Molitor, Hassan Harb, and Rita Streblow. Demand side management for city districts. Building and Environment (In Press), DOI: $\quad$ http://dx.doi.org/10.1016/j.buildenv.2015.03.026, $2015 . \quad$ ISSN 0360-1323. doi:http://dx.doi.org/10.1016/j.buildenv.2015.03.026. URL http://www.sciencedirect.com/science/ article/pii/s0360132315001432.

Christoph Nytsch-Geusen, Jörg Huber, Manuel Ljubijankic, and Jörg Rädler. Modelica buildingsystems - eine modellbibliothek zur simulation komplexer energietechnischer gebäudesysteme. Bauphysik, 35(1):21-29, 2013. ISSN 1437-0980. doi:10.1002/bapi.201310045. URL http: //dx.doi.org/10.1002/bapi.201310045.

Michael Wetter. Modelica Library for Building Heating, Ventilation and Air-Conditioning Systems. Lawrence Berkeley National Laboratory. Environmental Energy Technologies Division and Distributed by the Office of Scientific and Technical Information, U.S. Dept. of Energy, Berkeley and Calif and Oak Ridge and Tenn, 2009.

Michael Wetter. Modelica buildings library 2.0.0, 2015a. URL http://simulationresearch.lbl.gov/ modelica/FrontPage.

Michael Wetter. Buildings library user guide: 2. best practice, 2015b. URL http://simulationresearch.1bl. gov/modelica/userGuide/bestPractice. html\#thermo-fluid-systems.

Michael Wetter, Wangda Zuo, Thierry Stephane Nouidui, and Xiufeng Pang. Modelica Buildings library. Journal of Building Performance Simulation, 7(4):253-270, 2014. doi:10.1080/19401493.2013.765506.

Michael Wetter, Christoph van Treeck, and Jan Hensen. IEA EBC Annex 60, 2015. URL http://www. iea-annex60.org/index.html. 